Research Article

\title{
Small-Range High-Precision Positioning Based on Two-Point Coordination for Robot
}

\author{
Zhengping Li ${ }^{1},{ }^{1}$ Chaoliang Qin, ${ }^{2}$ and Hao Shi ${ }^{3}$ \\ ${ }^{1}$ North China University of Technology, Beijing 100144, China \\ ${ }^{2}$ Renesas Semiconductor Design (Beijing) Co., Ltd., Beijing 100085, China \\ ${ }^{3}$ The Ministry of Public Security of People's Republic of China, The First Research Institute, Beijing 100048, China \\ Correspondence should be addressed to Zhengping Li; lizp@ncut.edu.cn
}

Received 20 April 2021; Revised 15 May 2021; Accepted 24 May 2021; Published 16 June 2021

Academic Editor: Sang-Bing Tsai

Copyright (c) 2021 Zhengping Li et al. This is an open access article distributed under the Creative Commons Attribution License, which permits unrestricted use, distribution, and reproduction in any medium, provided the original work is properly cited.

This paper proposed a two-point coordinated positioning algorithm. Based on the assumption that the distance between two points was constant, a fusion algorithm was introduced into the positioning process to enhance the positioning accuracy. The simulation results showed that the proposed algorithm could reduce the RMS error to about 50\% of the improved sinc interpolation-based positioning algorithm when the sampling frequency was $500 \mathrm{MHz}$ and the interpolation number was 19.

\section{Introduction}

Accurate positioning is very important for robotic applications [1]. There are several kinds of indoor positioning systems. The non-radio-based technologies mainly employ cameras; the location accuracy is low when there are obstacles before the camera; and the image processing algorithm needs a high-speed processor that makes the cost and the power consumption very high. The commonly used approaches are radio-based technologies, such as Wi-Fi and ultra-wideband (UWB). Wi-Fi-based indoor positioning system primarily employs the strength of the Wi-Fi's access points (APs), and the accuracy is about $2 \mathrm{~m}$, which is too low to be used by many indoor applications [2-4]. UWB-based positioning system has high accuracy due to the large bandwidth, but because of the shadow fading and the random interferences, the positioning results are not stable. Moreover, time-based ranging technology was used in UWB positioning system, and several handshaking processes are needed to get the range between two nodes, which makes the positioning frequency low, and it cannot meet the requirements of some applications. Many research studies have been done on UWB-based localization [5-8] to enhance positioning stability. Some research studies employed filters to enhance the positioning accuracy [8], some research studies were focused on the ranging error elimination [5], and others attempted to fuse the data such as moving state and IMU data with the UWB positing data to eliminate the unstability $[6,7]$, and the problem is not solved yet. This paper proposed a two-point positioning data-fusing algorithm for the applications with more than one target nodes on an object [9].

This paper designed an improved positioning system that employed the proposed improved sinc interpolation algorithm to enhance the positioning frequency of the system for TDOA values. Then the system estimated the position of the target node using Chan's algorithm and used two-point coordination method to optimize the positioning results. As the result, we can reduce the sampling frequency to the maximum extent on the premise of ensuring accuracy.

The rest of the paper is organized as follows. Section 2 describes a survey of the related research studies; Section 3 describes the related principle of the proposed algorithm; Section 4 explains simulation and analysis of the algorithm; and Section 5 draws conclusion.

\section{Related Works}

With the rapid increase of data and multimedia services, the demand for positioning and navigation is increasing, especially in the complex indoor environment, such as the 
airport hall, exhibition hall, warehouse, supermarket, library, and underground parking lot.

TDOA (time difference of arrival) positioning is a kind of wireless positioning. By measuring the time of arrival of the signal to the reference node, the distance of the target node can be determined [10]. Using the distance between the target node and the various reference nodes, the location of the target node can be determined. However, the absolute time is difficult to measure; by comparing the time difference between the signals to the reference nodes, we can make the hyperbola with focus on the reference node and the long axis of the distance difference. The intersection of the hyperbolic is the location of the target node [11].

Based on TDOA, Li and Wang put forward a new algorithm that can greatly improve the positioning accuracy. Their system employs matched filter to calculate the TDOA value and does not need precise synchronization between the transmitter and receivers that makes the TDOA value more accurate [12]. In this paper, we propose the two-point coordination algorithm to improve data processing. The two-point coordination algorithm uses two-point information to calculate the position of the target node, while in the Small Range High Precision Positioning Algorithm Based on Improved Sinc only one-point information is used to calculate the position of the target node, so when we use twopoint coordination algorithm to process positioning results, it can improve the positioning accuracy.

\section{The Positioning Process and Related Algorithm}

3.1. The Positioning Process. The positioning steps are as follows:

Step 1: $L$ fixed target nodes received the FM wave signals from the reference node, of which $L$ was a positive integer. The modulation signal of the FM wave was a sawtooth signal, and therefore, the FM wave was called a sawtooth FM wave. A cycle of the sawtooth FM signal was called a chirp.

Step 2: we conducted amplitude limitation on the received signals, sampled on $M$ continuous chirps at interval $T$, and achieved the sample function $x_{i}(n)$ in which $i=1,2, \ldots, M$ and $n=0,1, \ldots, N$. $N$ was the sample point number of each chirp.

Step 3: by using improved sinc interpolation algorithm to reconstruct the sample function, we could get the reconstruction function $y(k)$ of which $k=0,1, \ldots$, $(N-1)(M+1)+M$.

Step 4: sampling the original sawtooth FM waves at interval $T /(M+1)$ and achieving the sample function $u(k)$, where $k=0,1, \ldots,(N-1)(M+1)+M$. Then, we took $u(k)$ and $y(k)$ to perform the cross-correlation operation and obtained the correlation peak location $A_{i}$ of which $i=0,1, \ldots, L$.

Step 5: using correlation peak location gap, we could calculate the signal arrival time difference $t_{2,1}, t_{3,1}, \ldots, t_{i, 1}, \ldots, t_{L, 1}$ between the $2^{\text {th }}, 3^{\text {th }}, \ldots, L^{\text {th }}$ reference node and the $1^{\text {th }}$ reference node. Among them $t_{2,1}, t_{3,1}, \ldots, t_{i, 1}, \ldots, t_{L, 1}$ were the TDOA values of which $t_{i, 1}=\left(A_{i}-A_{1}\right) * T$. Twas the time interval of the sample points in $y(k)$.

Step 6: the TDOA values and the coordinates of the reference nodes were put into Chan's algorithm to calculate the position of the target node.

Step 7: two-point coordination was used to optimize the positioning results.

3.2. The Related Algorithm. In Step 3, an improved sinc interpolation algorithm was mentioned, and a detailed description of the improved sinc interpolation algorithm was presented in the Small Range High Precision Positioning Algorithm Based on Improved Sinc Interpolation. In Step 6, the Chan's algorithm was mentioned and a detailed description of the Chan's algorithm was presented in the Precision Wireless Positioning Scheme in Small Range Based on First-Order Difference and Correlation Inspection. Therefore, we need not repeat the algorithm here.

In Step 7, the two-point coordination algorithm was mentioned, and a detailed description of the improved sinc interpolation algorithm was presented in this section.

It is assumed that the distance of two target nodes is known as $h$; the coordinates of the two target nodes $\left(x_{1}^{\prime}, y_{1}^{\prime}\right)$ and $\left(x_{2}^{\prime}, y_{2}^{\prime}\right)$ are estimated using the location algorithm.

If the distance between two points is greater than a certain distance, this set of data is considered to be a gross error and should be removed. Namely,

$$
h^{\prime}=\sqrt{\left(x_{1}^{\prime}-x_{2}^{\prime}\right)^{2}+\left(y_{1}^{\prime}-y_{2}^{\prime}\right)^{2}}>\omega h,
$$

where $\omega$ is an empirical value that is greater than 1 .

If $\left(x_{1}^{\prime}, y_{1}^{\prime}\right)$ and $\left(x_{2}^{\prime}, y_{2}^{\prime}\right)$ can be retained, according to $\left(x_{1}^{\prime}, y_{1}^{\prime}\right)$ and $\left(x_{2}^{\prime}, y_{2}^{\prime}\right)$, we estimate the location of the target node two times. For example, according to the coordinates of the target node $1\left(x_{1}^{\prime}, y_{1}^{\prime}\right)$ to estimate the coordinates of the target node $2\left(x_{2}^{\prime \prime}, y_{2}^{\prime \prime}\right)$, as the distance between two nodes $h$ is known, it is assumed that the target node $2\left(x_{2}^{\prime \prime}, y_{2}^{\prime \prime}\right)$ is in the circle with center point $\left(x_{1}^{\prime}, y_{1}^{\prime}\right)$ and a radius of $h$, and target node 2 is also in the straight line with the two points $\left(x_{1}^{\prime}, y_{1}^{\prime}\right)$ and $\left(x_{2}^{\prime}, y_{2}^{\prime}\right)$.

As we know there are two intersection points of a straight line and a circle, we choose the point that is closer to $\left(x_{2}^{\prime}, y_{2}^{\prime}\right)$ as the target node $2\left(x_{2}^{\prime \prime}, y_{2}^{\prime \prime}\right)$. We can estimate the $\left(x_{2}^{\prime \prime}, y_{2}^{\prime \prime}\right)$ according to the following equations:

$$
\left\{\begin{array}{l}
\sqrt{\left(x-x_{1}^{\prime}\right)^{2}+\left(y-y_{1}^{\prime}\right)^{2}}=h, \\
\frac{\left(x-x_{1}^{\prime}\right)}{\left(x_{2}^{\prime}-x_{1}^{\prime}\right)}=\frac{\left(y-y_{1}^{\prime}\right)}{\left(y_{2}^{\prime}-y_{1}^{\prime}\right)} .
\end{array}\right.
$$

There are two solutions to the equations, and we choose the final solution that is closer to $\left(x_{2}^{\prime}, y_{2}^{\prime}\right)$. The same can be used to solve the second-time estimation coordinates $\left(x_{1}^{\prime \prime}, y_{1}^{\prime \prime}\right)$ of the target node 1 . 
So far, the two estimation coordinates for each target node are obtained. Then the fusion algorithm is used to fuse the data of the two groups. The following is a method of calculating the weight.

Set the actual horizontal coordinates of the target node 1 $x$ :

$$
\begin{aligned}
& x_{1}^{\prime}=x+v_{1}, \\
& x_{1}^{\prime \prime}=x+v_{2} .
\end{aligned}
$$

where $v i(i=1,2)$ is a random error and $v i \sim N\left(0, \sigma_{i}^{2}\right)$, two observations are independent of each other.

It is assumed that the final estimation results $\hat{x}$ of $x$ are in linear relationship with the first estimate $x_{1}^{\prime}$ and the second estimate $x_{1}^{\prime \prime}$, and the $\hat{x}$ is the unbiased estimate of $x$ :

$$
\widehat{x}=\omega_{1} x_{1}^{\prime} \omega_{2} x_{1}^{\prime \prime} \text {. }
$$

where $\Omega=\left(\omega_{1}, \omega_{2}\right)$ is the weight value of the estimated value.

Set the estimation error:

$$
\tilde{x}=x-\widehat{x} .
$$

Take the cost function $\tilde{x}$ for the mean square error:

$$
J=E\left(\tilde{x}^{2}\right)=E\left\{\left[x-\omega_{1}\left(x+x_{1}^{\prime}\right)-\omega_{2}\left(x+x_{1}^{\prime \prime}\right)\right]^{2}\right\} .
$$

As the $\hat{x}$ is the unbiased estimate of $x$,

$$
E(\tilde{x})=E\left[x-\omega_{1}\left(x+x_{1}^{\prime}\right)-\omega_{2}\left(x+x_{1}^{\prime \prime}\right)\right]=0 .
$$

As $E\left(v_{1}\right)=E\left(v_{2}\right)=0$ and $E(x)=E(\widehat{x})$,

$$
\omega_{2}=1-\omega_{1} \text {. }
$$

Then the cost function can be written as

$$
J=E\left(\hat{x}^{2}\right)=E\left[\omega_{1}^{2} v_{1}^{2}+\left(1-\omega_{1}\right)^{2} v_{2}^{2}+2 \omega_{1}\left(1-\omega_{1}\right) v_{1} v_{2}\right]
$$

As $E\left(v_{1}^{2}\right)=\sigma_{1}^{2}$ and $E\left(v_{2}^{2}\right)=\sigma_{2}^{2}, v_{1}$ and $v_{2}$ are independent; $E\left(v_{1}, v_{2}\right)=0$.

Then,

$$
J=E\left(\tilde{x}^{2}\right)=\omega_{1}^{2} \sigma_{1}^{2}+\left(1-\omega_{1}\right)^{2} \sigma_{2}^{2} .
$$

In order to obtain the minimum value of $J$ and $\Omega$ derivatives,

$$
\frac{\partial J}{\partial \Omega}=0 .
$$

The optimal weight value is

$$
\begin{aligned}
& \omega_{1}^{*}=\frac{\sigma_{2}^{2}}{\sigma_{2}^{2}+\sigma_{1}^{2}}, \\
& \omega_{2}^{*}=\frac{\sigma_{1}^{2}}{\sigma_{2}^{2}+\sigma_{1}^{2}} .
\end{aligned}
$$

Optimal estimation is

$$
\widehat{x}=\frac{\sigma_{2}^{2} x_{1}^{\prime}}{\sigma_{2}^{2}+\sigma_{1}^{2}}+\frac{\sigma_{1}^{2} x_{1}^{\prime \prime}}{\sigma_{2}^{2}+\sigma_{1}^{2}} .
$$

In the same way, the vertical coordinates can also solved. The two-point coordination algorithm uses two-point information to calculate the position of the target node, while in the Small Range High Precision Positioning Algorithm Based on Improved Sinc only one-point information is used to calculate the position of the target node, so when we use two-point coordination algorithm to process positioning results, it can improve the positioning accuracy.

\section{System Simulation and Analysis}

In the simulation system, the positioning area was determined by the number of reference nodes, and the more the number of reference nodes, the larger the positioning area. The reference nodes were stationary, and they should be distributed around the positioning area uniformly as much as possible so that the system could get better positioning results.

In this simulation system, it supposes that the positioning range is $20 \mathrm{~m} \times 20 \mathrm{~m}$. The coordinates of seven reference nodes were $(0,0),(0,20),(10,-4),(20,0),(10,24)$, $(20,20)$, and $(-4,10)$. The target node acted as a transmitter. The modulation signal's frequency of the target node was $1 \mathrm{MHz}$. The simulation supposes that the transmission channel was 6-path Rician channel that had 1 line-of-sight (LOS) path and 5 reflection paths. The reflection paths were caused by the multipath effect because of the signal reflection, diffraction, and scattering. The additional delay of 6 paths were [0,31/1e9, 71/1e9, 109/1e9, 173/1e9, and 251/1e9] (s); the additional attenuation were $[0,-1,-9,-10,-15$, and $-20](\mathrm{db})$; and this was a common indoor channel. In addition, the received signal is summed together of the LOS and reflection signals. If the obstacles were on the LOS path, it should affect the TDOA value and cause TDOA errors.

The positioning accuracy was measured with the rootmean-square error (RMSE) of positioning results, which was frequently used at present (Figures 1-4). The positioning system simulation was done in different conditions through MATLAB (Tables 1-3):

(1) The positioning accuracy and time with different sinc interpolation algorithms are shown in Figure 1.

In Figure 1, abscissa was three different algorithms: the algorithm without any interpolation algorithm, the algorithm with nonimproved interpolation algorithm, and the algorithm with improved interpolation algorithm. The ordinates were RMSE and time. Table 1 lists the details of each point in Figure 1. The sampling frequency of the three algorithms in Figure 1 was $500 \mathrm{MHz}$, and the carrier frequency was $100 \mathrm{MHz}$. From the simulation results, it could be 


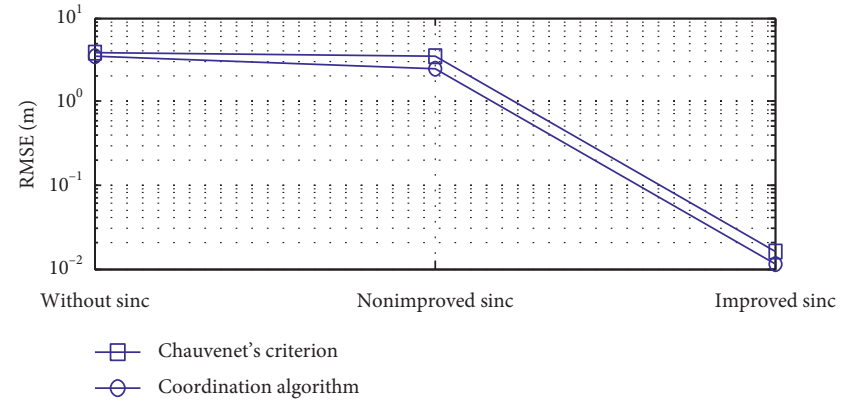

(a)

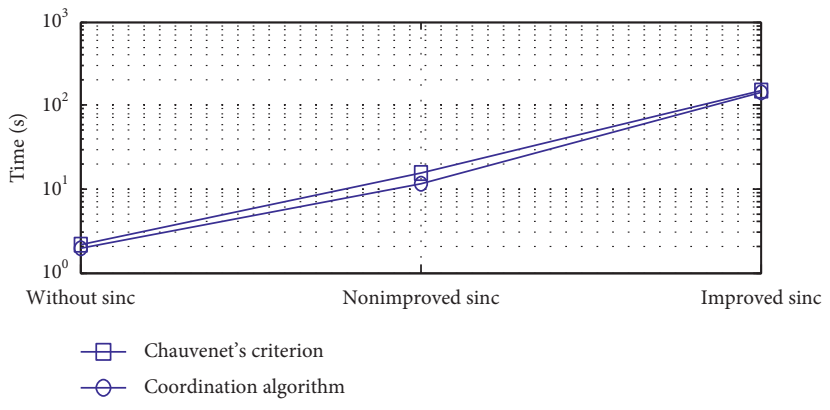

(b)

FIgURE 1: (a) RMSE and (b) time of different sinc interpolation algorithms.

TABLE 1: RMSE and time of different sinc interpolation algorithms.

\begin{tabular}{lcc}
\hline & RMSE square & RMSE circle \\
\hline & \multicolumn{3}{c}{ RMSE (m) } \\
Without sinc & 3.8979 & 3.4147 \\
Nonimproved sinc & 3.4813 & 2.8058 \\
Improved sinc & 0.0157 & 0.0142 \\
\hline \multicolumn{3}{c}{ Time (s) } \\
Without sinc & 2.1740 & 1.9254 \\
Nonimproved sinc & 15.1561 & 11.2563 \\
Improved sinc & 151.2365 & 143.2465 \\
\hline
\end{tabular}

seen that, in the process of data processing, the positioning accuracy and positioning time of the different degrees of improvement were compared between Chauvenet's criterion and coordination algorithm. When we do not use any interpolation algorithm, the algorithm has a lower positioning accuracy. Therefore, the positioning accuracy can be significantly improved when we use the coordination algorithm in the data processing. However, the positioning time of the algorithm is very short, so it is not obvious that the positioning time is shortened after we use the coordination algorithm $[13,14]$. When we use the improved interpolation algorithm, the algorithm has a lower positioning time. Therefore, the positioning time can be significantly improved when we use the coordination algorithm in the data processing. However, the positioning accuracy of the algorithm is very low, so it is not obvious that the positioning accuracy is promoted after we use the coordination algorithm. Then we will analyze the positioning accuracy in different situations in detail.

(2) The positioning accuracy in different sampling frequencies and interpolation points is shown in Figure 2:

In Figure 2, abscissa was carrier frequency, and the values were $10 \mathrm{MHz}, 20 \mathrm{MHz}, 30 \mathrm{MHz}, 40 \mathrm{MHz}$, $50 \mathrm{MHz}, 60 \mathrm{MHz}, 70 \mathrm{MHz}, 80 \mathrm{MHz}, 90 \mathrm{MHz}$, and $100 \mathrm{MHz}$. The ordinate was RMSE. Table 2 lists the details of each point in Figure 2. The target node was stationary, and it could be at any place in the positioning area. From the simulation results, it could

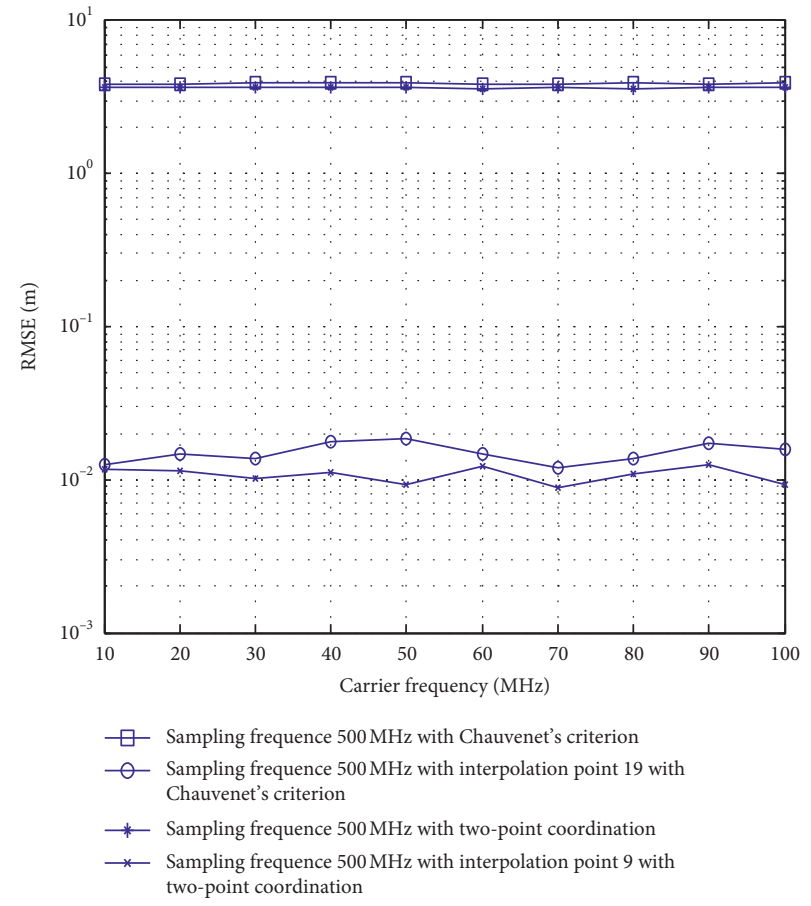

FIGURE 2: RMSE of different algorithms in different sampling frequencies and interpolation points.

TABLE 2: RMSE of different algorithms in different sampling frequencies and interpolation points.

\begin{tabular}{lcccc}
\hline \multirow{2}{*}{$\begin{array}{l}\text { RF } \\
(\mathrm{MHz})\end{array}$} & RMSE & RMSE & RMSE & RMSE cross \\
& square & circle & asterisk & \\
\hline 10 & 3.8295 & 0.0125 & 3.6916 & 0.0118 \\
20 & 3.8724 & 0.0148 & 3.6835 & 0.0113 \\
30 & 3.8928 & 0.0107 & 3.6557 & 0.0102 \\
40 & 3.8995 & 0.0176 & 3.6531 & 0.0112 \\
50 & 3.8983 & 0.0184 & 3.6459 & 0.0104 \\
60 & 3.8196 & 0.0148 & 3.6184 & 0.0121 \\
70 & 3.8812 & 0.0119 & 3.6357 & 0.0089 \\
80 & 3.8886 & 0.0138 & 3.6198 & 0.0109 \\
90 & 3.8700 & 0.0174 & 3.6525 & 0.0124 \\
100 & 3.8979 & 0.0157 & 3.6554 & 0.0093 \\
\hline
\end{tabular}


be seen that the algorithm with improved sinc interpolation comparing with the algorithm without improved sinc interpolation in positioning accuracy had a very large enhancement. The RMSE decreased from about $3 \mathrm{~m}$ to about $0.01 \mathrm{~m}$. From the simulation results, it could be seen that the algorithm with improved sinc interpolation with two-point coordination compared with the algorithm with improved sinc interpolation with Chauvenet's criterion in positioning accuracy had certain enhancement. The RMSE decreased from about $0.015 \mathrm{~m}$ to about $0.010 \mathrm{~m}$. We could also see from the results that the carrier frequency had little influence on the positioning accuracy when the carrier frequency varies from $10 \mathrm{MHz}$ to $100 \mathrm{MHz}$.

(3) The positioning accuracy in different numbers of interpolation points is shown in Figure 3.

In Figure 3, abscissa was the numbers of interpolation points, and the values were 0,9 and 19. The ordinate was RMSE. We set the carrier frequency to $50 \mathrm{MHz}$. Table 3 lists the details of each point in Figure 3. From the simulation results, it could be seen that when there were no interpolation points, whether the Chauvenet's criterion or two-point coordination is used, the positioning accuracy is relatively low. The RMSE is over $3.0 \mathrm{~m}$. When we interpolated 9 points to the $500 \mathrm{MHz} / 1 \mathrm{GHz}$ sampling chips, the positioning accuracy improved obviously. The algorithm with two-point coordination compared with the algorithm with Chauvenet's criterion in positioning accuracy had certain enhancement. Since the positioning accuracy of the sampling frequency with 9 interpolation points to $1 \mathrm{GHz}$ sampling chips was enough high, there was little accuracy improvement when we interpolated 19 points to the $1 \mathrm{GHz}$ sampling chips.

(4) The positioning accuracy in different sampling frequencies is shown in Figure 4.

In Figure 4, abscissa was the sampling frequency, and the values were $250 \mathrm{MHz}, 500 \mathrm{MHz}$, and $1000 \mathrm{MHz}(1 \mathrm{GHz})$. The ordinate was RMSE (m). We set the carrier frequency to $50 \mathrm{MHz}$. Table 4 lists the details of each point in Figure 4. From the simulation results, it could be seen that the positioning accuracy was not high in all three sampling frequencies when there were no interpolation points, whether the Chauvenet's criterion or two-point coordination is used. The accuracy of the $250 \mathrm{MHz}$ sampling frequency with $9 / 19$ interpolation points was close to that of the $2.5 \mathrm{GHz} / 5 \mathrm{GHz}$ sampling frequency without interpolation points. When the positioning accuracy is relatively low, the effect of using twopoint coordination to improve the positioning accuracy is obviously compared with the Chauvenet's criterion. When the accuracy is over about $3.0 \mathrm{~m}$, using two-point coordination can improve the positioning accuracy by about $0.2 \mathrm{~m}-0.3 \mathrm{~m}$. Due to the improved sinc algorithm, the positioning accuracy improves obviously. The effect of using two-point coordination to improve the positioning accuracy

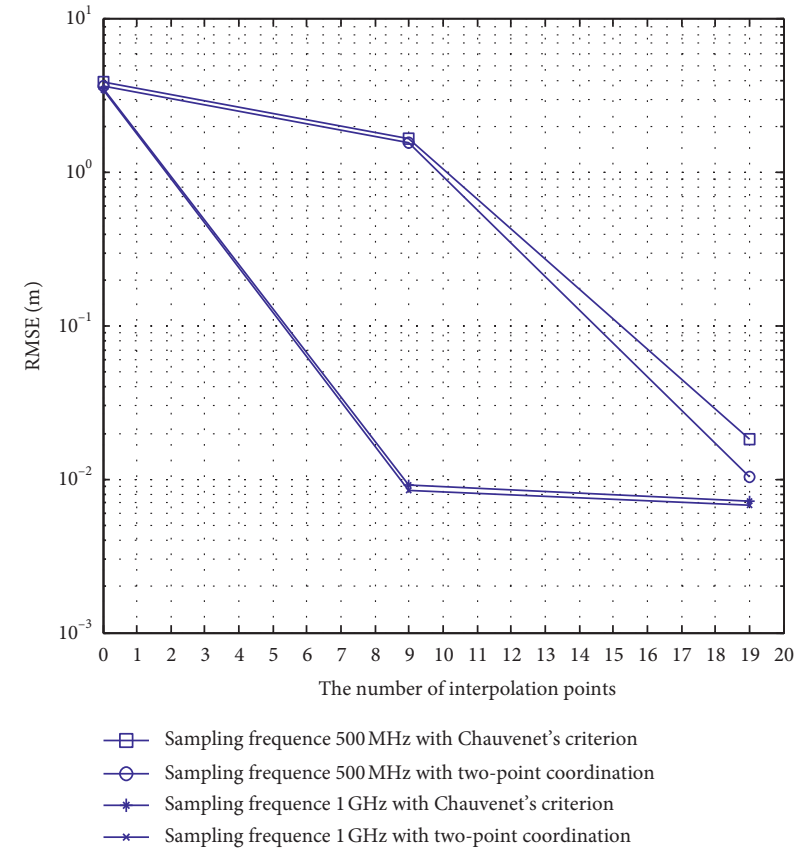

FIGURE 3: RMSE of different algorithms in different numbers of interpolation points.

TABLE 3: RMSE of different algorithms in different numbers of interpolation points.

\begin{tabular}{lccc}
\hline \multirow{2}{*}{ RMSE (m) } & \multicolumn{3}{c}{ Interpolation numbers } \\
& 0 & 9 & 19 \\
\hline RMSE (square) & 3.8983 & 1.6520 & 0.0184 \\
RMSE (circle) & 3.6459 & 1.5649 & 0.0104 \\
RMSE (asterisk) & 3.5430 & 0.0092 & 0.0072 \\
RMSE (cross) & 3.4758 & 0.0084 & 0.0068 \\
\hline
\end{tabular}

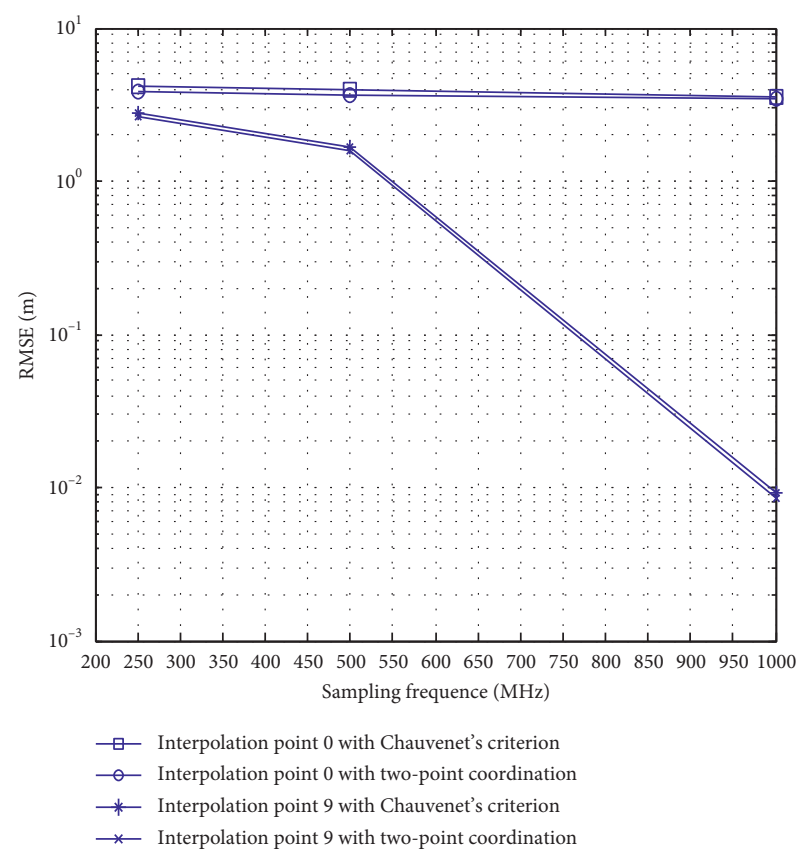

FIgURE 4: RMSE of different algorithms in different numbers of interpolation points. 
TABLE 4: RMSE of different algorithms in different sampling frequencies.

\begin{tabular}{lccc}
\hline RMSE (m) & \multicolumn{3}{c}{ SF (MHz) } \\
& 250 & 500 & 1000 \\
\hline RMSE (square) & 4.1463 & 3.8983 & 3.5430 \\
RMSE (circle) & 3.8172 & 3.6459 & 3.4758 \\
RMSE (asterisk) & 2.8120 & 1.6520 & 0.0092 \\
RMSE (cross) & 2.6274 & 1.5649 & 0.0084 \\
\hline
\end{tabular}

is not obvious comparing with the Chauvenet's criterion. When the accuracy is under $1.0 \mathrm{~m}$, using two-point coordination can improve the positioning accuracy by about $0.001 \mathrm{~m}-0.003 \mathrm{~m}$.

The positioning accuracy and positioning time of the have different degrees of improvement compared between Chauvenet's criterion, which is used in the Small Range High Precision Positioning Algorithm Based on Improved Sinc and coordination algorithm.

\section{Conclusion}

This paper introduces the present situation and the future development of the wireless location, summarizes the related technologies and algorithms, and proposes a coordination localization algorithm. The analysis and simulation results show that if the coordination algorithm is used in the data processing, it can improve the positioning accuracy of the system. The primary contribution was that a two-point coordination algorithm is proposed that could greatly increase positioning accuracy when the sampling frequency was low.

The problem is that when we use the improved sinc interpolation positioning algorithm, the positioning accuracy can meet the requirements, but we have to wait for a certain amount of time to form a new chip to calculate the TDOA values even though we use the coordination algorithm to optimize the positioning time. And when there were more than one target nodes in the positioning area, it would take longer to estimate a position. The next work will be to continue to study the relationship between positioning accuracy and positioning time.

\section{Data Availability}

The data that support the findings of this study are available from the corresponding author upon reasonable request.

\section{Conflicts of Interest}

The authors declare that they have no conflicts of interest.

\section{Acknowledgments}

This paper was supported by the National Key Research and Development Program (Program ID: 2020YFC0811004).

\section{References}

[1] X. Chen, Z. Liu, J. Wan, and Z. Li, “Aggregated handover authentication for machine type communication," Journal of Organizational and End User Computing, vol. 31, no. 3, pp. 83-96, 2019.

[2] Y. Tao and L. Zhao, "A novel system for WiFi radio map automatic adaptation and indoor positioning," IEEE Transactions on Vehicular Technology, vol. 67, no. 11, pp. 1-10, 2018.

[3] J. W. Jang and S.-N. Hong, "Indoor localization with WiFi fingerprinting using convolutional neural network," in Proceedings of the 10th International Conference on Ubiquitous and Future Networks, pp. 753-758, Prague, Czech Republic, July 2018.

[4] W. Sun, M. Xue, H. Yu, H. Tang, and A. Lin, "Augmentation of fingerprints for indoor WiFi localization based on Gaussian process regression," IEEE Transactions on Vehicular Technology, vol. 67, no. 11, pp. 10896-10905, 2018.

[5] M. Stampa, M. Mueller, D. Hess, and C. Roehrig, "Semiautomatic calibration of UWB range measurements for an autonomous mobile robot," in Proceedings of the 50th International Symposium on Robotics (ISR 2018), pp. 300-305, Munich, Germany, June 2018.

[6] T.-M. Nguyen, A. H. Zaini, C. Wang, K. Guo, and L. Xie, "Robust target-relative location with ultra-wideband ranging and communication," in Proceedings of the 2018 International Conference on Robotics and Automation (ICRA 2018), pp. 2312-2319, Brisbane, QLD, Australia, September 2018.

[7] C. Pierre, R. Chapuis, R. Aufrère, J. Laneurit, and C. Debain, "Rang-only based cooperative localization for mobile robots," in Proceedings of the 21st International Conference on Information Fusion (FUSION), pp. 1933-1939, Cambridge, UK, July 2018.

[8] Z. Kasmi, N. Guerchali, A. Norrdine, and J. H. Schiller, "Algorithms and position optimization for a decentralized localization platform based on resource-constrained devices," Journal of IEEE Transactions on Mobile Computing, vol. 18, no. 8, pp. 1731-1744, 2018.

[9] Y. Huang, W. Sheng, P. Jin, B. Nie, M. Qiu, and G. Xu, “A node-oriented discrete event scheduling algorithm based on finite resource model," Journal of Organizational and End User Computing, vol. 31, no. 3, pp. 67-82, 2019.

[10] C.-L. Wei and C.-T. Ho, "Exploring signaling roles of service providers' reputation and competence in influencing perceptions of service quality and outsourcing intentions," Journal of Organizational and End User Computing, vol. 31, no. 1, pp. 86-109, 2019.

[11] Y. Yu, Y. Yao, and X. Cheng, "TDOA positioning technology and practical application," China Radio, vol. 11, pp. 57-58, 2014.

[12] Z. Li, Z. Wang, Y. Zhang, and L. Ma, "Precision wireless positioning scheme in small range based on first-order difference and correlation inspection," Journal of Information Technology Research, vol. 6, no. 3, pp. 1-15, 2013.

[13] M. Zhou, Y. Wang, Y. Liu, and Z. Tian, "An informationtheoretic view of WLAN localization error bound in GPSdenied environment," IEEE Transactions on Vehicular Technology, vol. 68, no. 4, pp. 4089-4093, 2019.

[14] M. Zhou, X. Li, Y. Wang, S. Li, Y. Ding, and W. Nie, "6G multi-source information fusion based indoor positioning via Gaussian kernel density estimation," IEEE Internet of Things Journal, vol. 10, no. 99, p. 1, 2020. 